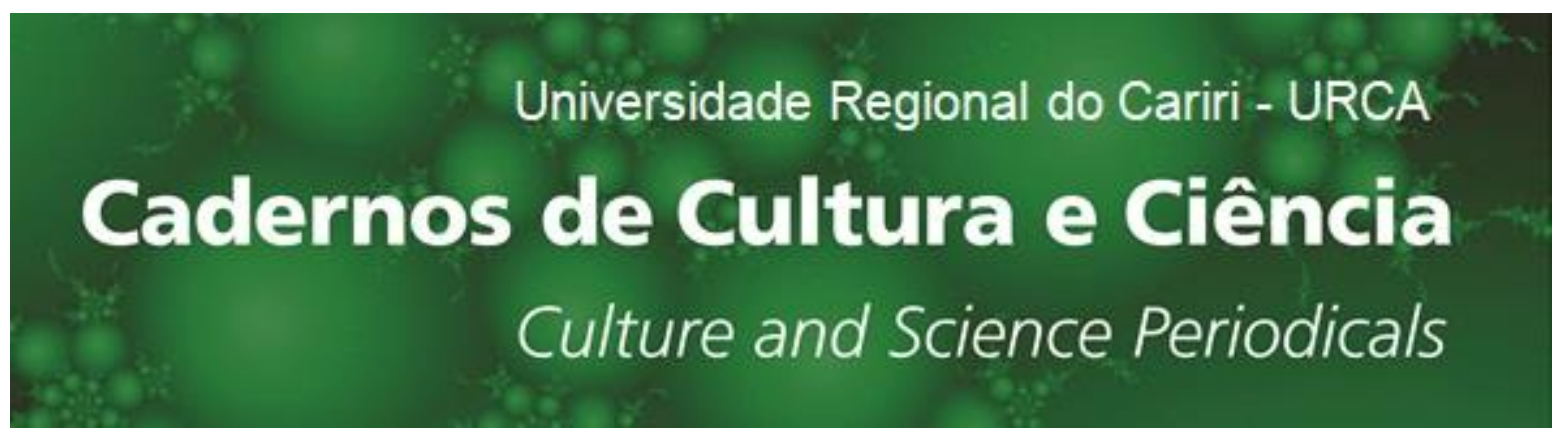

DOI: $10.14295 /$ cad.cult.cienc.v18i1.2422

\title{
DIA ROSA: UM RELATO DE EXPERIÊNCIA
}

Eny Ribeiro de Lemos ${ }^{1}$; Ilary Gondim Dias Sousa ${ }^{2}$; Herla Pereira Gonçalves ${ }^{3}$; Izabelly Barbosa Lima Soares ${ }^{3}$; Rafaela Moraes Guedes 3 ; Márcia Ferraz Pinto ${ }^{4}$

Resumo: A falta de acesso a informações é um dos problemas que mais leva ao adoecimento das mulheres. A participação em projetos na comunidade adstrita é importante na formação de um médico humanizado. Além disso, propagar informações essenciais para melhorar a qualidade de vida feminina. No dia 18 de outubro de 2016, um grupo de estudantes de medicina conduziu uma roda de conversa sobre câncer de colo de útero e câncer de mama em uma unidade de saúde localizada no Geisel, João Pessoa-PB, com mulheres que aguardavam atendimento. A preparação para essa ação foi realizada no mês anterior, no qual foi confeccionado panfletos, materiais decorativos, cartazes, quizzes e selecionados brindes para quem acertasse perguntas. A experiência trouxe benefícios tanto para o aprendizado acadêmico como para a população daquela unidade de saúde. Na ação foram escutados os anseios e dúvidas sobre os assuntos abordados e utilizamos ferramentas lúdicas buscando sempre a participação ativa das mulheres. Na abordagem do autoexame das mamas, utilizou-se modelos anatômicos para facilitar a demonstração da técnica e encorajar que as mulheres treinassem. Assim, foi possível o esclarecimento dos questionamentos e enfatizar a importância da prevenção. Ocorreu uma importante participação dos ouvintes o que possibilitou a aprendizagem da dinâmica da unidade e a importância da educação em saúde no cuidado integral da população. Do mesmo modo, as mulheres partilharam suas experiências e aprenderam sobre as técnicas necessárias para detecção precoce do câncer. A ação trouxe um importante aprendizado para a vida de todos os envolvidos. Por isso, novos projetos devem ser estimulados.

Palavras-chave: Saúde da Mulher. Estratégia de Saúde da Família. Promoção da Saúde.

1. Acadêmica de Medicina da Universidade de Fortaleza (Unifor), Fortaleza, Ceará, Brasil;

2. Acadêmica de Medicina da Universidade Federal de Campina Grande - Campus Cajazeiras, Cajazeiras, Paraíba, Brasil; 


\title{
PINK DAY: AN EXPERIENCE REPORT
}

\begin{abstract}
Lack of access to information is one of the problems that most leads to women's illness. Participation in community projects is important in training a humanized physician. Besides that propagating essential information to improve women's quality of life. On October 18, 2016, a group of medical students conducted a discussion round about cervical and breast cancer at the Basic Health Care Unit, located in Geisel, João Pessoa-PB, with women. The preparation for this action was carried out in the previous month: pamphlets, decorative materials, letters, quizzes and gifts selected for those who answered the questions were carefully made. The experience provided benefits for both academic and population learning. In the action, the students cleared the doubts and used ludic tools in order to encourage the active participation of women. For the explanation of the breast self-examination, there were employed anatomical models to facilitate demonstration of the technique. Thus, it was possible to focus on technique, clarify questions and emphasize the importance of prevention. There was an important participation of listeners that enabled students to learn about the unit's dynamic and the importance of health education in the integral care of the population. The women shared their experiences and learned about the necessary techniques to detect cancer in an early stage. The action brought important learning into the lives of everyone involved. Therefore, new projects must be stimulated.
\end{abstract}

Keywords: Women's Health. Family Health Strategy. Health promotion.

\section{Introdução}

O câncer de mama é uma doença prevalente na população feminina brasileira e são estimados 59.700 casos novos apenas para 2019, o que simboliza um risco estimado de 56,33 casos a cada 100 mil mulheres (BRASIL, 2017). Ela tem uma etiologia multifatorial e vários fatores contribuem para o aumento do risco individual de desenvolvimento da doença.

Os principais fatores são idade entre 40 a 60 anos e presença de familiares de primeiro grau com a doença. A esses somam-se a ingestão de bebidas alcoólicas, a nuliparidade, o sedentarismo e não-amamentação. A existência de um ou mais desses fatores na vida de uma paciente, torna necessário o maior cuidado com o aparecimento de sinais típicos da doença, como dor na mama e alterações na pele da mesma (SILVA; RIUL, 2011; PINHO; COUTINHO, 2005).

O câncer de colo de útero, por sua vez, é o quarto tipo de câncer mais comum nas mulheres brasileiras, ficando atrás apenas dos cânceres de pele não melanoma, de mama e de cólon. Para ele, estimam-se 16.370 casos novos em 2019 e um risco estimado de 15,43 casos a cada 100 mil mulheres (BRASIL, 2017).

O principal fator de risco é a infecção pelo vírus HPV e a faixa etária mais acometida vai 
dos 25 aos 60 anos, sendo que estudos mostram que exames preventivos periódicos podem reduzir em até $70 \%$ a sua mortalidade na população de risco. Além disso, essa doença tem vários sinais característicos e que permitem um diagnóstico precoce, como sangramento ou corrimento vaginal incomuns e dispareunia (dor durante as relações sexuais) (CESAR et. al., 2003).

Diante do que foi exposto, é possível perceber a facilidade do paciente em se identificar com parte de um grupo de risco ou mesmo com portador de sinais das doenças. No entanto, principalmente devido à falta de conhecimento, muitas mulheres, não conseguem reconhecer os sinais e sintomas que podem ser relevante para um diagnóstico ágil.

A falta de acesso a informações é um dos problemas que mais leva ao adoecimento na população feminina. Isso se relaciona diretamente com o acompanhamento preventivo e tratamento precoce de doenças como os cânceres de colo de útero e de mama que têm maior chance de cura quanto mais cedo detectadas (SILVA et. al., 2014).

O impacto do nível de informação na precocidade do diagnóstico foi apresentado por Brenna et al. (2001). Em estudo realizado em um centro de ginecologia da cidade de São Paulo, eles mostraram que 63\% das mulheres entrevistadas com câncer de colo de útero invasivo (um estágio mais avançado do câncer) não sabiam a utilidade do Papanicolau, exame recomendado para detecção precoce da doença.

Sendo assim, alunas de Medicina de João Pessoa, Paraíba decidiram aproveitar o Outubro Rosa para realizar uma Mega Ação em uma unidade básica de saúde local visando a ensinar as mulheres usuárias da unidade os fatores de risco, os métodos de prevenção e os principais sintomas dos cânceres de mama e de colo de útero.

\section{Metodologia}

Trata-se de trabalho científico do tipo relato de experiência. Ele foi baseado em rodas de conversa realizadas no Outubro Rosa de 2016 em uma Unidade Básica de Saúde no bairro Geisel, João Pessoa -PB por estudantes do curso de medicina.

Os temas adotados para as rodas de conversa entre as mulheres foram câncer de mama e de colo uterino, sendo estes escolhidos devido ao período do Outubro Rosa e à relevância que a informação tem na detecção precoce dessa doenças. Após a escolha dos temas, foi realizado um planejamento baseado no número de pessoas que estariam presentes na ação e feitos convites para 
população que utiliza o PSF, a serem distribuídos por meio dos agentes comunitários de saúde.

Foram confeccionados panfletos e cartazes informativos sobre os temas abordados para poder orientar a roda de conversa. Para tornar a ação ainda mais dinâmica, a Unidade foi decorada antes do início das atividades com o tema de Outubro Rosa e foram feitas perguntas ao final da ação para saber se o conhecimento havia sido sedimentado e as dúvidas sanadas, ocorrendo a entrega de prêmios (kits de higiene pessoal) para as participantes que acertassem. Para as mulheres que foram com seus filhos, foi reservada uma sala na unidade para que as crianças ficassem, sob cuidado de estudantes, durante a ação.

Todos os assuntos foram abordados de forma bastante profissional e com a seriedade e importância que necessitavam, tentando criar sempre um ambiente que deixasse as mulheres à vontade e seguras para participarem, envolvendo-se nas demonstrações e expondo seus medos e questionamentos. Todos os assuntos foram expostos da forma mais simples e prática possível. Para a explicação do autoexame da mama, por exemplo, foram utilizados modelos anatômicos para demonstrar de forma mais clara sua feitura e achados patológicos e encorajar as mulheres a treinar sem que se sentissem intimidadas.

Além disso, para incentivar ainda mais a participação das ouvintes e fazer com que elas ficassem até o final da ação, foram distribuídos lanches e cédulas contendo pontuação (5, 10 e 50) para os que respondiam as perguntas, davam algum depoimento ou tiravam dúvidas. Esses pontos, no final da ação poderiam ser trocados por roupas num bazar organizado na frente da UBS pelos estudantes.

As peças do bazar foram doadas pelas estudantes como também por seus familiares. Essa metodologia animou o público alvo a participar e transmitir os conhecimentos adquiridos.

\section{Resultados e Discussão}

A educação em saúde é de fundamental importância na prevenção e no diagnóstico precoce das diferentes patologias, como o câncer. Nesse sentido, quando se aborda o câncer, especificamente o de mama e o de colo de útero, é necessário atentar para relevância dos exames preventivos para detecção precoce do agravo e para o tratamento adequado (INCA, 2018a).

O câncer de mama é uma patologia causada pela multiplicação de células anormais da mama, que foram o tumor. Segundo o INCA (2018b), Há vários tipos de câncer de mama. Alguns tipos têm desenvolvimento rápido enquanto outros são mais lentos. 
Vale salientar que a maioria dos casos tem boa resposta ao tratamento, principalmente quando diagnosticado e tratado no início (BRASIL, 2014).

Outra perspectiva importante é que um em cada três casos de câncer pode ser curado se for descoberto logo no início. Porém, muitas pacientes, por medo ou desconhecimento, preferem não abordar o assunto e acabam atrasando o diagnóstico (BRASIL, 2014).

Diante disso, é de vital importância, estimular ações que visam a educação e saúde, como mecanismo de conscientização e compreensão da importância da prevenção do câncer de mama.

As ações de prevenção ajudam a minimizar o custo de cuidado com saúde, além de melhorar a qualidade de vida das pessoas (RODRIGUES; CRUZ; PAIXÃO, 2015).

A maior consciência da mulher sobre detecção precoce do câncer de mama será mais efetiva se entendida como parte de um conjunto de ações que, simultaneamente, possam assegurar a disponibilidade de rede assistencial e profissionais capacitados a atuantes para diagnosticar e tratar as lesões identificadas em prazo adequado (SILVA et al., 2014).

Para a detecção precoce do câncer de mama são utilizadas técnicas de exame clínico das mamas e a mamografia como exame complementar de padrão ouro (RODRIGUES; CRUZ; PAIXÃO, 2015).

Com relação ao câncer de colo de útero, existe uma fase pré-clínica (sem sintomas) do câncer do colo do útero, em que a detecção de lesões precursoras (que antecedem o aparecimento da doença) pode ser feita através do exame preventivo (Papanicolau). Quando diagnosticado na fase inicial, as chances de cura do câncer cervical são de 100\%. Conforme a evolução da doença, aparecem sintomas como sangramento vaginal, corrimento e dor (CARVALHO; DWER; RODRIGUES, 2018).

O exame preventivo (Papanicolau) é a principal maneira para o diagnóstico da doença. É de vital importância que os serviços de saúde orientem sobre o que é e qual a importância da realização periódica permite diminuir a mortalidade da doença. Indiscutivelmente, o principal fator de risco para o desenvolvimento do câncer do colo do útero é a infecção pelo papilomavírus humano (HPV), que é a infecção viral mais comum do aparelho reprodutor feminino e masculino (CARVALHO; DWER; RODRIGUES, 2018; GAMA, 2018).

Portanto, é de vital importância, ações que visem propagar e sedimentar o conhecimento para que ocorra um melhor acompanhamento, prevenção e diagnóstico precoce das pacientes. 


\section{Conclusão}

O câncer de colo de útero e de mama são os únicos que possuem rastreamento, e quando diagnosticado precocemente aumenta a taxa de sobrevida da paciente, logo é de fundamental importância a educação em saúde para que as mulheres saibam da importância do rastreamento e da prevenção. Além disso, é necessário respeitar a cultura da paciente, informar a importância da mamografia e do Papanicolau, detalhar sobre o tratamento, tudo isso para conscientizar e quebrar possíveis preconceitos que possam existir.

Este contexto permite perceber a importância da ações educativas, pois esta ação permitiu passar conhecimentos científicos de forma prática, lúdica e interativa, permitindo que as usuárias do PSF em que foi realizada percebessem a importância da prevenção e foi capaz de sanar suas dúvidas e inseguranças em relação ao câncer de colo de útero e de mama. Além disso a iniciativa de distribuir cédulas para que as pacientes pudessem participar posteriormente do bazar foi de fundamental importância para garantir que a roda de conversa acontecesse de forma ativa e com a participação de uma grande quantidade de mulheres, garantindo assim que o conhecimento passado fosse sedimentado e entendido por elas. Assim, essa ação trouxe um importante aprendizado para a vida de todos os envolvidos

Com isso, foi observado que é necessário que as próprias unidades de saúde incentivem a realização periódica deste rastreamento, passando para os pacientes a importância dessa prevenção, pois falta conhecimento por parte da população em geral. Portanto, é de vital importância, ações que visem propagar e sedimentar o conhecimento para que ocorra um melhor acompanhamento, prevenção e diagnóstico precoce das pacientes. E esse trabalho deve ser feito de forma multiprofissional, garantindo que todos os profissionais da unidade de saúde propaguem o máximo de conhecimento possível, garantindo assim um atendimento integral e humanizado.

\section{Referências}

BRASIL. Ministério da Saúde. Estimativa 2018: Incidência de Câncer no Brasil. 1 ed. Rio de Janeiro: INCA, 2017. 128 p.

BRASIL. Ministério da Saúde. Instituto Nacional de Câncer José Alencar Gomes da Silva. Câncer de mama: é preciso falar. 1 ed. Rio de Janeiro: INCA, 2014. 16 p.

BRENNA, S. M. et al. Conhecimento, atitude e prática do exame de Papanicolau em mulheres 
com câncer de colo uterino. Cadernos em Saúde Pública. v. 17, n. 4, p. 909-914, 2001.

CARVALHO, P.; DWER, G.; RODRIGUES, N. Trajetórias assistenciais de mulheres entre diagnóstico e início de tratamento do câncer de colo uterino. Saúde Debate. v. 42, n. 118, p. 687701,2018

CESAR, J. A. et al. Fatores associados à não realização de exame citopatológico de colo uterino no extremo Sul do Brasil. Cadernos em Saúde Pública. v. 19, n. 5, n. 1365-1372, 2003.

GAMA, G. A importância da prevenção do câncer de colo de útero. Revista Saúde Premium. Disponível em: <http://revista.saudepremium.com/2018/02/13/a-importancia-da-prevencao-docancer-de-colo-de-utero/>. Acesso em: 22 nov. 2018.

INCA. Detecção Precoce (2018a). Disponível em: <https://www.inca.gov.br/controle-do-cancerdo-colo-do-utero/acoes-de-controle/deteccao-precoce>. Acesso: 22 nov. 2018

INCA. Câncer de mama. (2018 b). Disponível em: <https://www.inca.gov.br/tipos-decancer/cancer-de-mama>. Acesso: 22 nov. 2018.

PINHO, V. F.; COUTINHO, E. S. Risk factors for breast cancer: a systematic review of studies with female samples among the general population in Brazil. Cadernos em Saúde Pública. v. 21, n. 2, p. 351-360, 2005.

RODRIGUES, J. D.; CRUZ, M. S.; PAIXÃO, A. N. Uma análise da prevenção do câncer de mama no Brasil. Revista Ciências \& Saúde Coletiva. v. 20, n. 10, p. 3163-3176, 2015.

SILVA, G. et al. Acesso à detecção precoce do câncer de mama no Sistema Único de Saúde: uma análise a partir dos dados do Sistema de Informações em Saúde. Cadernos em Saúde Pública. v. 30, n. 7, p. 1537-1550, 2014.

SILVA, P. A.; RIUL, S. S. Câncer de mama: fatores de risco e detecção precoce. Revista Brasileira de Enfermagem. v. 64, n. 6, p. 1016-1021, 2011.

Submetido: 10 dezembro 2018

Aceito: 29 julho 2019 